\title{
EFEITOS METABÓLICOS DA L-ALANIL-GLUTAMINA EM RATOS SUBMETIDOS À ISQUEMIA DA PATA TRASEIRA ESQUERDA SEGUIDA DE REPERFUSÃO ${ }^{1}$
}

\author{
João Martins de Souza Torres² \\ Sérgio Botelho Guimarães ${ }^{3}$ \\ Paulo Roberto Leitão de Vasconcelos ${ }^{4}$ \\ Maria Cecília Resende Martins ${ }^{5}$ \\ Carolyne Rolim Chaves ${ }^{5}$ \\ Paulo Roberto Cavalcante de Vasconcelos ${ }^{5}$
}

\begin{abstract}
Torres JMS, Guimarães SB, Vasconcelos PRL, Martins MCR, Chaves CR, Vasconcelos PRC. Efeitos metabólicos da l-alanil-glutamina em ratos submetidos à isquemia da pata traseira esquerda seguida de reperfusão. Acta Cir Bras [serial online] 2003 Jan-Fev;18(1). Disponível em URL: http://www.scielo.br/acb.
\end{abstract}

RESUMO - Objetivo: Objetivou-se investigar os efeitos metabólicos da L-alanil glutamina no tecido muscular e sangue arterial de ratos Wistar submetidos à isquemia aguda da pata traseira. Métodos: Utilizaram-se 48 ratos machos distribuídos em 4 grupos (1- controle / 2 - experimento), redistribuídos em 2 subgrupos (n=06). Trinta minutos após a injeção de uma solução a $20 \%$ de Lalanil-glutamina $(0,75 \mathrm{mg}$ / grupo 2$)$ ou solução salina (grupo 1) na veia jugular direita ocluiu-se a artéria ilíaca comum esquerda por 30 minutos, por pinçamento. Amostras musculares e de sangue arterial foram obtidas logo após a remoção da pinça (tempo 0) e 5, 15 e 30 mais tarde. Resultados: Observou-se redução significativa $(\mathrm{p}<0,05)$ da concentração de glicose tissular no animal tratado 15 minutos após o início da reperfusão. Houve aumento significativo das concentrações de glicose (grupo 2) nos tempos $15 \mathrm{~min}$ e $30 \mathrm{~min}$ em relação ao tempo $0 \mathrm{~min}$. Não houve diferença na glicemia entre os dois grupos. Estes achados sugerem uma maior utilização da glicose pelo músculo isquêmico, através do ciclo malato-aspartato. A ausência de diferenças entre as concentrações de piruvato tissular, comparando-se os 2 grupos poderia ser explicada pelo reduzido tempo de isquemia. Conclusão: A L-alanil-glutamina estimula a. maior utilização da glicose, via glicólise, pela ativação do ciclo lactato-malato.

DESCRITORES - Músculo. Isquemia. Lesão de reperfusão. L-alanil-glutamina. Ratos.

\section{INTRODUÇÃO}

Conceitua-se isquemia como o desequilíbrio maior ou menor entre a oferta e demanda de sangue para um determinado órgão ou setor do corpo, provocando correspondente hipóxia tissular com todas as suas conseqüências patogênicas, de acordo com a intensidade e duração da mesma.

1. Trabalho realizado no Laboratório de Cirurgia Experimental (LABCEX), Programa de Pós-Graduação stricto sensu em Cirurgia, Faculdade de Medicina, Departamento de Cirurgia da Universidade Federal do Ceará (UFC).

2. Professor Adjunto de Cirurgia e Mestrando em Cirurgia Experimental do Departamento de Cirurgia da UFC.

3. Mestre em Cirurgia, Professor Adjunto do Departamento de Cirurgia, Vice-Coordenador de Graduação da UFC.

4. Professor Doutor $(P h D)$, Coordenador do Curso de Pós-Graduação stricto sensu em Cirurgia da Faculdade de Medicina da UFC

5. Estudantes de Medicina da UFC. 
A doença vascular é o substrato patogênico principal e final da isquemia. Dados epidemiológicos apontam repetidamente a doença cardiovascular como a principal "causa mortis" e também um fator importante de incapacitação na maioria dos países industrializados $^{1}$. Nos Estados Unidos ela foi responsável por aproximadamente $44 \%$ de todos os óbitos, em 1987, vindo em segundo lugar o câncer com metade deste percentual ${ }^{2}$.

Há de se distinguir formas agudas e crônicas de isquemia, assim como se Reveste-se de fundamental importância o conceito de isquemia fria e quente. A isquemia fria, bem conduzida, protege significativamente os tecidos, especialmente se associadas a soluções nutrientes geladas, como as usadas nos transplantes cardíaco, renal e hepático, afora as diversas soluções cardioplégicas, usadas em grande escala, na cirurgia cardíaca com parada anóxica ${ }^{3,4}$. Por outro lado, na isquemia quente, na dependência de sua duração, ocorre uma incapacidade de retorno do fluxo sangüíneo quando a circulação é refeita.

A glutamina é um aminoácido condicionalmente essencial, pois se torna indispensável nas condições de estresse, sendo ativamente transportada e metabolizada em quase todos os tecidos animais ${ }^{5}$. O presente trabalho, utiliza a L-alanil-glutamina (um dipeptídeo precursor da glutamina) como eventual opção terapêutica coadjuvante no tecido isquêmico. A existência de poucos registros na literatura especializada, estudando essa forma de utilização, motivou esta pesquisa.

Objetivou-se, neste trabalho estudar eventuais alterações induzidas por L-alanil-glutamina nas concentrações in vivo sangüíneas e tissulares dos metabólitos (lactato, piruvato, glicose e corpos cetôni$\cos$ ) em ratos submetidos à isquemia muscular esquelética, seguida de reperfusão.

\section{MÉTODOS}

A pesquisa foi realizada de acordo com as Normas Internacionais para a Pesquisa Biomédica em Animais (1990) e de acordo com a Lei Federal $\mathrm{n}^{\circ} .6 .638$, de 08 de maio de 1979. ${ }^{6}$ Utilizaram-se 48 ratos albinos (Rattus norvegicus albinus), da linhagem Wistar, machos, com peso oscilando entre 300 e 350 gramas, provenientes do biotério central da Universidade Federal do Ceará e mantidos no Laboratório de Cirurgia Experimental do Departamento de Cirurgia da Faculdade de Medicina (UFC) em gaiolas de aço, recebendo água e alimento adequado ad libitum até 12 horas antes do início do experimento. Os ratos foram distribuídos eqüitativamente em dois grupos: Grupo 1 (controle) e Grupo 2 (experimento). Cada grupo foi subdividido em dois subgrupos $(\mathrm{n}=6)$. Todos os procedimentos cirúrgicos foram realizados sob anestesia geral inalatória, com éter dietílico. Após canulação da veia jugular injetava-se uma solução de L-alanil-glutamina a $20 \%$, na proporção de $0,75 \mathrm{~g} / \mathrm{kg}$ corporal (Dipetiven $\mathbb{\circledR}$, Laboratório Frenesisus) nos animais do grupo experimento (G-2) e mesmo volume de solução salina nos animais do grupo controle (G-1). Após 30 minutos a artéria ilíaca comum esquerda era ocluída por pinçamento com pinça vascular oclusiva, tipo buldogue. Confirmava-se a eficiência do bloqueio pela ausência dos pulsos arteriais, hipotermia e cianose da musculatura da coxa e o uso do fluxômetro Doppler Passados 30 minutos retirava-se a pinça e se confirmava o retorno do fluxo arterial pelos sinais clínicos e o uso do fluxômetro Doppler. Fragmentos musculares foram retirados da coxa esquerda bem com $2,0 \mathrm{ml}$ de sangue arterial ao término do período de isquemia e 5,15 e 30 minutos mais tarde, para análise enzimática.

Os metabólitos no sangue da aorta e no tecido muscular da coxa esquerda (PE) foram determinados por métodos de ensaio enzimático ${ }^{7,89}$. Foram incluídos neste experimento os precursores da gliconeogênese (piruvato e lactato), os corpos cetônicos (acetoacetato e 3-hidroxibutirato) e a D-glicose. As concentrações dos metabólitos foram calculadas em $\mu \mathrm{mol} / \mathrm{g}$ tecido fresco ou sangue.

Os resultados foram expressos como média \pm E.P.M. (Erro Padrão da Média). O teste não paramétrico de Mann-Whitney foi utilizado para a análise estatística, fixando-se o valor de significância em 5\% $(\mathrm{p}<0,05)$.

\section{RESULTADOS}

Analisando-se as concentrações de glicose na pata esquerda dos animais tratados com L-alanil-glutamina, observou-se redução significativa da concentração de glicose tissular em comparação às concentrações do metabólito na pata esquerda do animal não tratado (controle), 15 minutos após o início da reperfusão. Por outro lado, comparando-se as concentrações de glicose, nos diversos tempos de reperfusão com o tempo 0 min, observou-se aumento significativo das concentrações de glicose nos tempos $15 \mathrm{~min}$ e $30 \mathrm{~min}$. (Figura 1). 


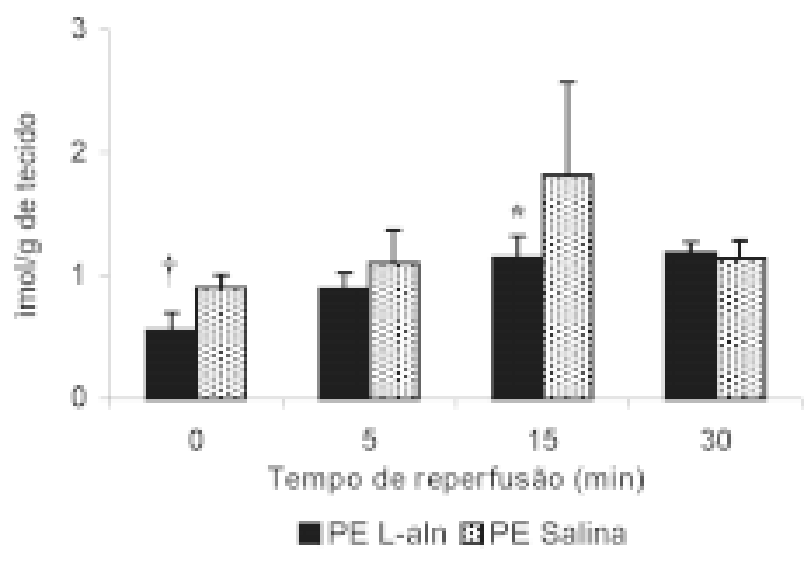

* $\mathrm{p}<0,05$ comparado a PE Salina do grupo $15 \mathrm{~min}$ $\uparrow \mathrm{p}<0,05$ comparado a PE L-aln dos grupos 15 e 30

(Teste de Mann-Whitney)

FIGURA 1 - Concentração de glicose no músculo (pata esquerda traseira).

Não foram observadas alterações significantes da glicemia em nenhum dos grupos estudados (Figura 2).

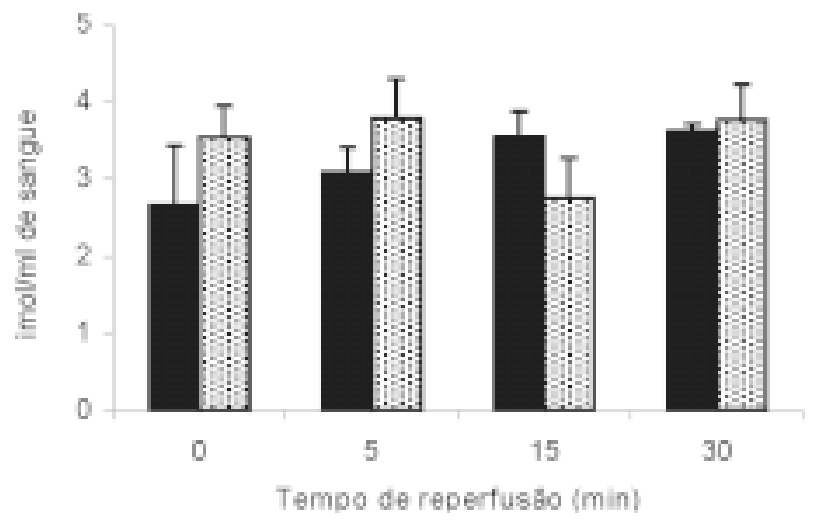

a Sangue L-aln 『Sangue Salina

(Teste de Mann-Whitney)

FIGURA 2 - Concentração de glicose no sangue

Não foram observadas diferenças nas concentrações tissulares de piruvato entre os grupos tratados e não tratados. Observou-se, no entanto, uma queda significante da concentração de piruvato no músculo durante a reperfusão, nos tempos 5, 15 e 30 minutos, em relação à concentração do metabólito no tempo 0 min, em ambos os grupos (Figura 3).

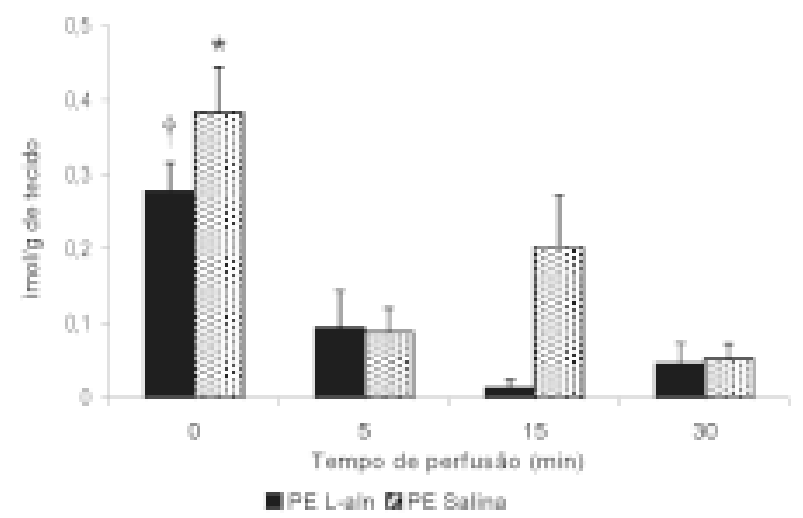

* $\mathrm{p}<0,05$ comparado a PE Salina dos grupos 5 e $30 \mathrm{~min}$

$\uparrow \mathrm{p}<0,05$ comparado a PE L-aln dos grupos 5,15 e $30 \mathrm{~min}$

(Teste de Mann-Whitney)

FIGURA 3 - Concentração de piruvato no músculo (pata esquerda traseira)

Não foram observadas diferenças significantes da concentração de piruvato no sangue, comparando-se os animais tratados com os não tratados. Observou-se uma queda significante da concentração de piruvato, no sangue dos animais não tratados, nos diversos tempos estudados, em relação ao tempo $0 \mathrm{~min}$. (Figura 4).

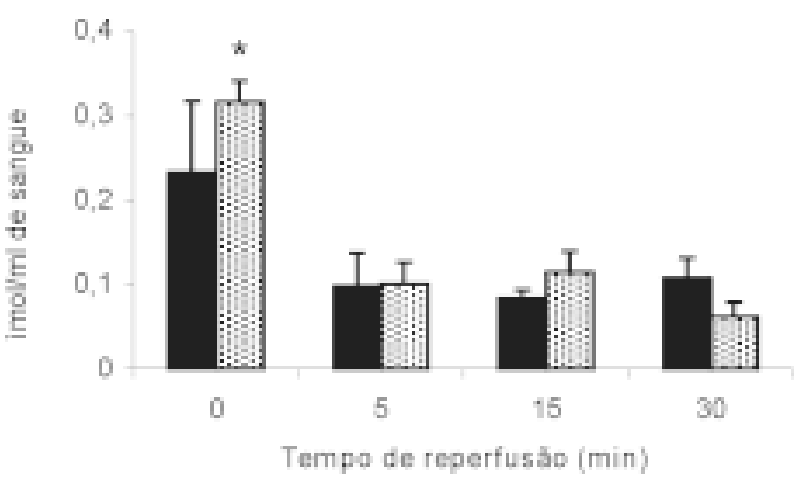

Sangue L-aln $\boldsymbol{Z}$ Sangue Salina

* p $<0,05$ - grupo 0 min comparado aos grupos 5 , 15 e $30 \mathrm{~min}$

(Teste de Mann-Whitney)

FIGURA 4 - Concentração de piruvato no sangue.

Não foram observadas diferenças, comparandose as concentrações de lactato no músculo dos animais 
tratados e não tratados. Houve queda significante da concentração de lactado aos 15 minutos de reperfusão em relação ao tempo 5 minutos, no tecido muscular dos animais do grupo experimento (Figura 5).

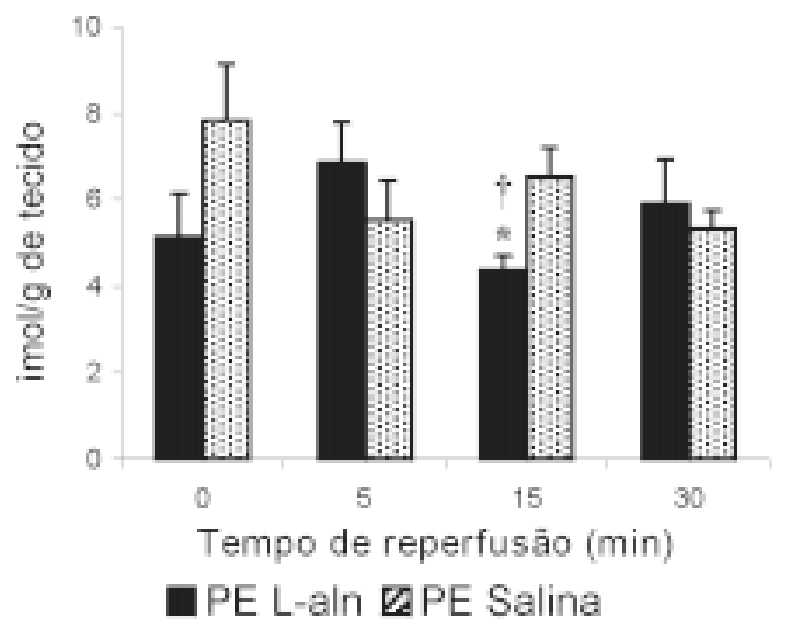

* $\mathrm{p}<0,05$ comparado a PE Salina do grupo $15 \mathrm{~min}$ $\uparrow \mathrm{p}<0,05$ comparado a PE L-aln do grupo $5 \mathrm{~min}$

(Teste de Mann-Whitney)

FIGURA 5 - Concentração de lactato (músculo).

Houve queda com significância estatística na concentração de lactato, aos 5 minutos de reperfusão, no sangue dos animais não tratados comparada à concentração lactato no sangue dos animais previamente tratados com com L-alanil-glutamina (Figura 6).

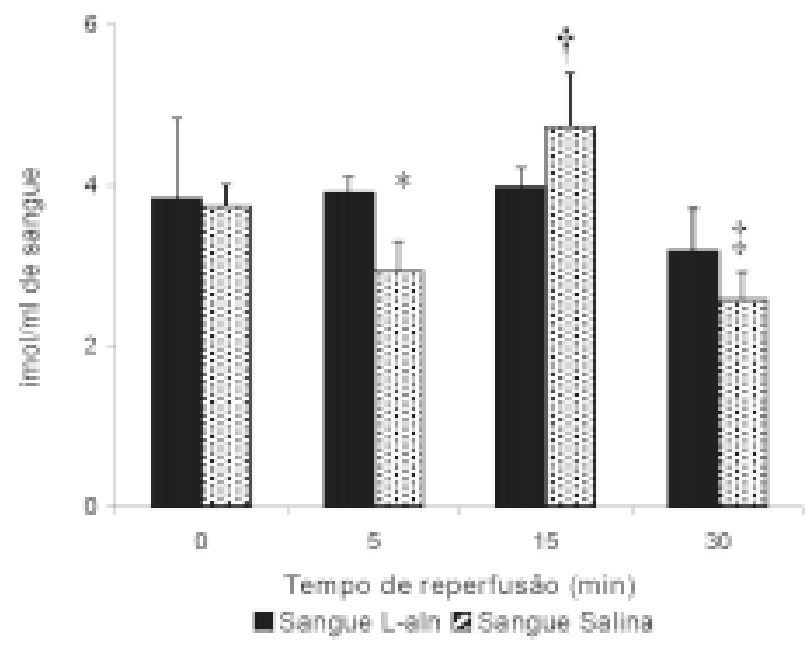

$* \mathrm{p}<0,05$ comparado a Sangue Salina do grupo $5 \mathrm{~min}$ $\uparrow \mathrm{p}<0,05$ comparado a Sangue Salina dos grupos 5 e $30 \mathrm{~min}$ $\$ \mathrm{p}<0,05$ comparado a Sangue Salina dos grupos 0 e $5 \mathrm{~min}$

(Teste de Mann-Whitney)

FIGURA 6 - Concentração de lactato (sangue).
Observou-se redução da concentração de corpos cetônicos no tecido muscular da pata traseira dos ratos tratados com L-alanil-glutamina no tempo $5 \mathrm{~min}$ em relação aos animais não tratados. $\mathrm{O}$ estudo das concentrações de corpos cetônicos tissulares nos animais previamente tratados evidenciou um aumento significante das concentrações destes substratos aos 30 minutos de início da reperfusão em relação aos valores aferidos nos tempos 0 e $5 \mathrm{~min}$. (Figura 7). Observou-se, no sangue, diminuição estatisticamente significante da concentração de corpos cetônicos nos tempos 5 e 15 minutos, em relação ao tempo 0 de reperfusão (Figura 8).

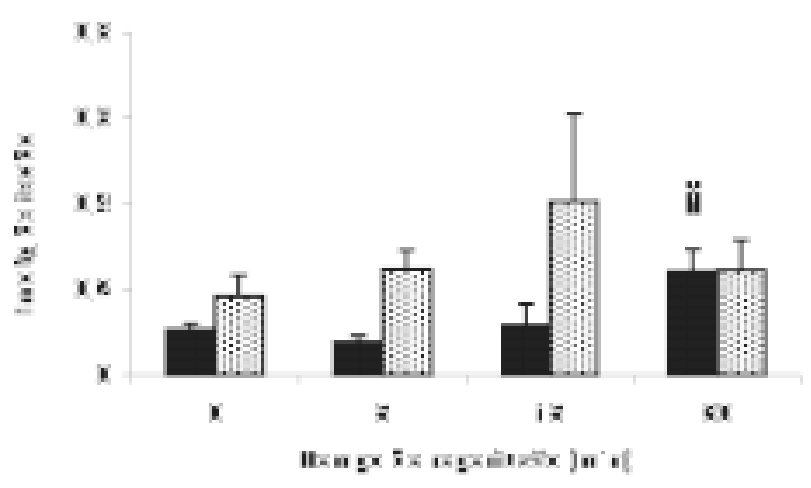

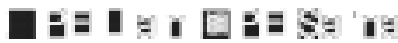

* $\mathrm{p}<0,05$ comparado a PE Salina do grupo $5 \mathrm{~min}$

$\uparrow \mathrm{p}<0,05$ comparado a PE L-aln dos grupos 0 e 5 min

(Teste de Mann-Whitney)

FIGURA 7 - Concentração de corpos cetônicos no músculo.

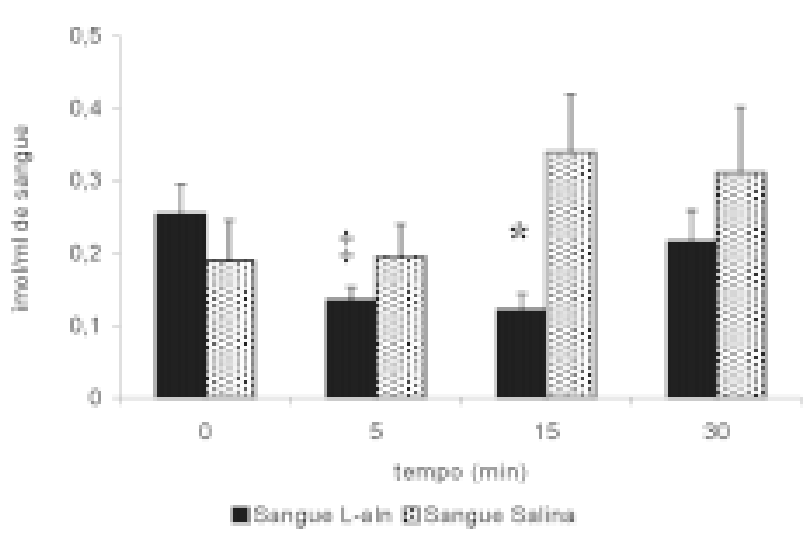

$* \mathrm{p}<0,05$ comparado a Sangue Salina do grupo $15 \mathrm{~min}$
$\uparrow \mathrm{p}<0,05$ comparado a Sangue L-aln do grupo $0 \mathrm{~min}$
$+\mathrm{p}<0,05$ comparado a Sangue L-aln do grupo 0 min
(Teste de Mann-Whitney)

FIGURA 8 - Concentração de corpos cetônicos no sangue. 


\section{DISCUSSÃO}

No presente estudo, a redução significante $(\mathrm{p}<$ $0,05)$ da concentração tissular de glicose na pata esquerda dos animais tratados com L-alanil-glutamina com relação à concentração aferida nos animais não tratados (Figura 1) pode estar relacionada a uma maior utilização da glicose (glicólise aumentada). Este aumento na eficiência da queima de glicose em tecido isquêmico, rico em glutamina exógena, sugere: 1) captação e transformação de glutamina em glutamato pela enzima glutaminase, ativada durante a hipóxia tissular; 2) a maior eficiência da via glicolítica pode dever-se a uma ativação do ciclo lactato-malato, resultante da maior disponibilidade de glutamato para o grupo isquêmico de animais recipientes de L-alanilglutamina ${ }^{10,11}$.

O músculo esquelético é um tecido eminentemente produtor de glutamina, sendo rico em glutaminasintetase. Neste estudo houve oferta exógena de glutamina (L-alanil-glutamina), que é facilmente utilizada por tecidos ricos em glutaminase, tais como as hemácias, a medula renal e o intestino delgado ${ }^{12}$. Pesquisadores demonstraram, em situações de estresse hipóxico, atividade aumentada de glutaminase no músculo esquelético de ratos submetidos a 7, 14 e 21 dias de hipóxia, através de simulação de altitude $(7620 \mathrm{~m})$ em câmara hipobárica ${ }^{13}$. Portanto, é de se esperar que, no presente estudo, o tecido muscular esquelético, submetido a 30 minutos de isquemia (oclusão arterial seletiva) possa vir a apresentar atividade aumentada da referida enzima. A ausência de alterações na glicemia pode ser explicada pela atividade reguladora do fígado.

A ausência de diferenças entre as concentrações de piruvato tissular, comparando-se os animais tratados com os ratos que receberam solução salina na reperfusão poderia ser explicada pelo reduzido tempo de isquemia. Por outro lado, a queda da concentração de piruvato nos diversos tempos de reperfusão, em ambos os grupos, em relação aos valores aferidos no tempo 0 minuto era esperada, pois esse metabólito, por desempenhar um papel central nas vias de transformação metabólica, apresenta níveis fugazes de concentração em seus diversos tempos de reperfusão ${ }^{14}$. Explica-se a queda do piruvato, nos animais tratados, 15 minutos após o início da reperfusão, pela ação da L-alanilglutamina, que estimularia a conversão desse metabólito em lactato.

A diminuição da concentração de lactato tissular, após 15 minutos de reperfusão, nos animais tratados, sugere um efeito benéfico da L-alanil-glutamina, pela ativação do ciclo lactato-malato. $\mathrm{O}$ mesmo se aplica à queda das concentrações de corpos cetônicos, com maior utilização desse substrato, para a produção de energia, 5 minutos após o início da reperfusão.

\section{CONCLUSÃO}

A redução da concentração de glicose no tecido muscular isquêmico nos animais que receberam L-alanilglutamina sugere maior utilização da glicose, via glicólise, pela ativação do ciclo lactato-malato,

\section{REFERÊNCIAS}

1. Giannine SD. In: Aterosclerose: Dislipidemias. 1ed. São Paulo: BG Cultural; 1999. p 2-5.

2. Luepker RV. In: Compêndio de cardiologia preventiva. Editora de Publicações Científicas Ltda; 1994. p 1-10.

3. Kirklin JW, Barrat-Boyes BG. In: Cardiac surgery. 2ed. New York: Ed. Churchill and Living-Stone; 1993. p 129-65.

4. Biberthaler P, Luchting B, Massberg S, Teupser D, Langer S, Leiderer R, Krombach F, Messmer K. Ischemia at 4 degrees C: a novel model to investigate the effect of hypothermia on postischemia hepatic microcirculatory injury. Res Exp Med (Berl) 2001; 200 (2): 93-105.

5. Smith MD. Glutamine metabolism and its physiologic importance. J Parent Ent Nutr 1990; 14: 40-4.

6. Brasil. Lei Federal n. 6.638, de 8 de maio de 1979. Estabelece normas para a prática didático-científica da vivissecção de animais e determina outras providências. In Brasil. Coleção de leis de 1979: atos legislativos do poder executivo. Leis de abril a junho. Brasília, Departamento de Imprensa Nacional, 1979. p 33-4.

7. Hohorst apud Vasconcelos PRL. Hepatic metabolism during sepsis [Tese - Doutorado]. University Oxford; 1987.

8. Williamson DH, Mellanby J, Krebs HA. Enzymic determinations of the D-(-) $\beta$-hydroxybutyric acid and acetoacetic acid in blood. Biochem J 1962; 82: 90-6.

9. Slein apud Vasconcelos PRL. Hepatic metabolism during sepsis. [Tese - Doutorado]. University Oxford; 1987.

10. Newcomb R, Pierce AR, Kano T, Meng W, Bosque-Hamilton P, Taylor L, Curthoys N, Lo EH. Characterization of mitochondrial glutaminase and amino cids at prolonged times after experimental focal cerebral ischemia. Brain Res 1998; 813: $103-11$.

11. Khogali SE, Harper AA, Lyall JA, Rennie MJ. Effects of Lglutamine on post-ischaemic cardiac function: protection and rescue. J Moll Cel Cardiol 1998; 30: 819-27.

12. Souba WW, Smith RJ, Wilmore DW. Glutamine metabolism by intestinal tract. J Parent Ent Nutr 1985; 9:608-17.

13. Vats P, Mukherjee AK, Kumria MM, Singh SN, Patil SK, Rangnathan S, Sridharan K. Changes in the activity levels of glutamine synthetase, glutamine and glycagon synthetase in rats subjected to hypoxic stress. Int J Biometereol 1999; 42(4):205-9.

14. Lehninger AL. Glicólise: a via central do catabolismo da glicose In: Princípios de bioquímica. São Paulo: Sarvier; 1984. p.288310 
Torres JMS, Guimarães SB, Vasconcelos PRL, Martins MCR, Chaves CR, Vasconcelos PRC. Metabolic effects of 1-alanyl-glutamine in rats subjected to left hind leg ischemia and reperfusion. Acta Cir Bras [serial online] 2003 Jan-Feb;18(1). Available from URL: http://www.scielo.br/acb.

ABSTRACT - Purpose: An experimental study has been conducted to investigate acute ischemic changes in concentrations of glucose, piruvate, lactate and ketone bodies in rat hind leg muscle and arterial blood after intravenous injection of l-alanil-glutamine solution. Methods: Forty-eight male Wistar rats were distributed into two groups: Group 1 (Control) and Group 2 (Experiment). Each group was divided into 4 subgroups $(n=6)$. Thirty minutes after administration of $20 \%$ solution of L-alanil-glutamine $(0,75 \mathrm{~g} / \mathrm{Kg}$, group 1$)$ or saline solution (group 2) into right jugular vein, a clamp was placed across left common iliac artery for 30 minutes. Samples from left thigh muscle and arterial blood from abdominal aorta were collected at the end of ischemic period (subgroup 0) and 5,15 and 30 minutes later. Results: Glucose concentrations were decreased $(p<0.05)$ in experiment group muscles, compared to respective control (subgroup $15 \mathrm{~min}$ ) and significantly increased in subgroups 15 and $30 \mathrm{~min}$ as compared to subgroup $0 \mathrm{~min}$. There was no difference in glycemia and pyruvate concentrations when comparing experiment versus control rats. Decreased lactate concentrations might be related to L-alanyl glutamine effects. Conclusions: Increased availability of L-alanil-glutamine increases energy production via glycolysis by activation of lactate-malate cycle in ischemic muscle tissue.

KEY WORDS - Muscle. Ischemia. Reperfusion lesion. L-alanyl-glutamine. Rats.

Conflito de interesse: nenhum

Fonte de financiamento: nenhuma

Correspondência:

João Martins de Souza Torres

Departamento de Cirurgia

Rua Prof. Costa Mendes, 1608/ $3^{\circ}$ andar

60430-140 Fortaleza - Ceará

Tel: (85) 288-8063

Fax: (85) 288-8064

mcirur@npd.ufc.br

Data do recebimento: $18 / 10 / 2002$

Data da revisão: 03/11/2002

Data da aprovação: 15/11/2002 